\title{
Experimental validation of a novel smart electromechanical tuned mass damper beam device
}

\author{
S. Rafique ${ }^{\text {a }}$, P. Bonello ${ }^{\mathrm{a}, *}$, R. Shuttleworth ${ }^{\mathrm{b}}$ \\ a School of Mechanical, Aerospace and Civil Engineering, University of Manchester, Manchester M13 9PL, UK \\ ${ }^{\mathrm{b}}$ School of Electrical and Electronic Engineering, University of Manchester, Manchester M13 9PL, UK
}

\section{A R T I C L E I N F O}

\section{Article history:}

Received 26 November 2012

Received in revised form

2 April 2013

Accepted 24 April 2013

Handling Editor: H. Ouyang

Available online 29 May 2013

\begin{abstract}
A B S T R A C T
This paper validates the novel concept of utilising piezoelectric vibration energy harvesting (PVEH) beams as a tuned mass damper (TMD)-which suppresses a particular vibration mode of a generic host structure over a broad band of excitation frequencies. The proposed device comprises a pair of bimorphs shunted by a resistor, capacitor and inductor connected in various alternative circuit configurations. A benchmark for the performance is established through Den Hartog's theory for the optimal damping of a classical TMD. Experimental results demonstrate that such optimal damping is equivalently generated by the PVEH effect for appropriately tuned circuitry. These results correlate reasonably well with the results of a theoretical analysis introduced in a previous paper. The proposed TMD beam device combines the relative advantages of the classical ('mechanical') TMD and the shunted piezoelectric patch ('electrical' vibration absorber), presenting the prospect of a functionally more readily-adaptable class of 'electromechanical' tuned vibration absorbers. Moreover, with further development, this dual PVEH/TMD beam device holds the potential of simultaneous energy storage.
\end{abstract}

(c) 2013 Elsevier Ltd. All rights reserved.

\section{Introduction}

A tuned vibration absorber (TVA), in its most generic form, is an auxiliary system whose parameters can be tuned to suppress the vibration of a host structure. The auxiliary system is commonly a structure that is equivalent to a spring-massdamper system, in which case the TVA is referred to here as a 'mechanical' TVA. This device can be used in two distinct ways, resulting in different optimal tuning criteria and design requirements [1]: (a) as a vibration neutraliser (undamped vibration absorber) [2,3]; (b) as a tuned mass damper (TMD) [4]. The present paper is concerned with the latter application, wherein the device is used to suppress (dampen) the vibration contributed by a specific troublesome natural mode of frequency $\Omega_{T}$ of the host structure over a wide band of excitation frequencies $\omega$. Following the classical theory of Den Hartog $[1,4]$, apart from the optimal tuning of the device's frequency $\omega_{a}$, the TMD requires the prescription of an optimal level of damping.

As discussed in [5], the practical implementation of the correct amount of damping in a TMD makes its design more challenging relative to the neutraliser and, once implemented, such damping may be difficult to adjust in response to a variation in the system parameters. The requirement for damping means that simple, compact and readily tuneable beamlike designs, that are popular with neutralisers [2,3], are relatively difficult to realise for TMDs. Beam-like (cantilever-type)

\footnotetext{
* Corresponding author.

E-mail address: philip.bonello@manchester.ac.uk (P. Bonello).
} 


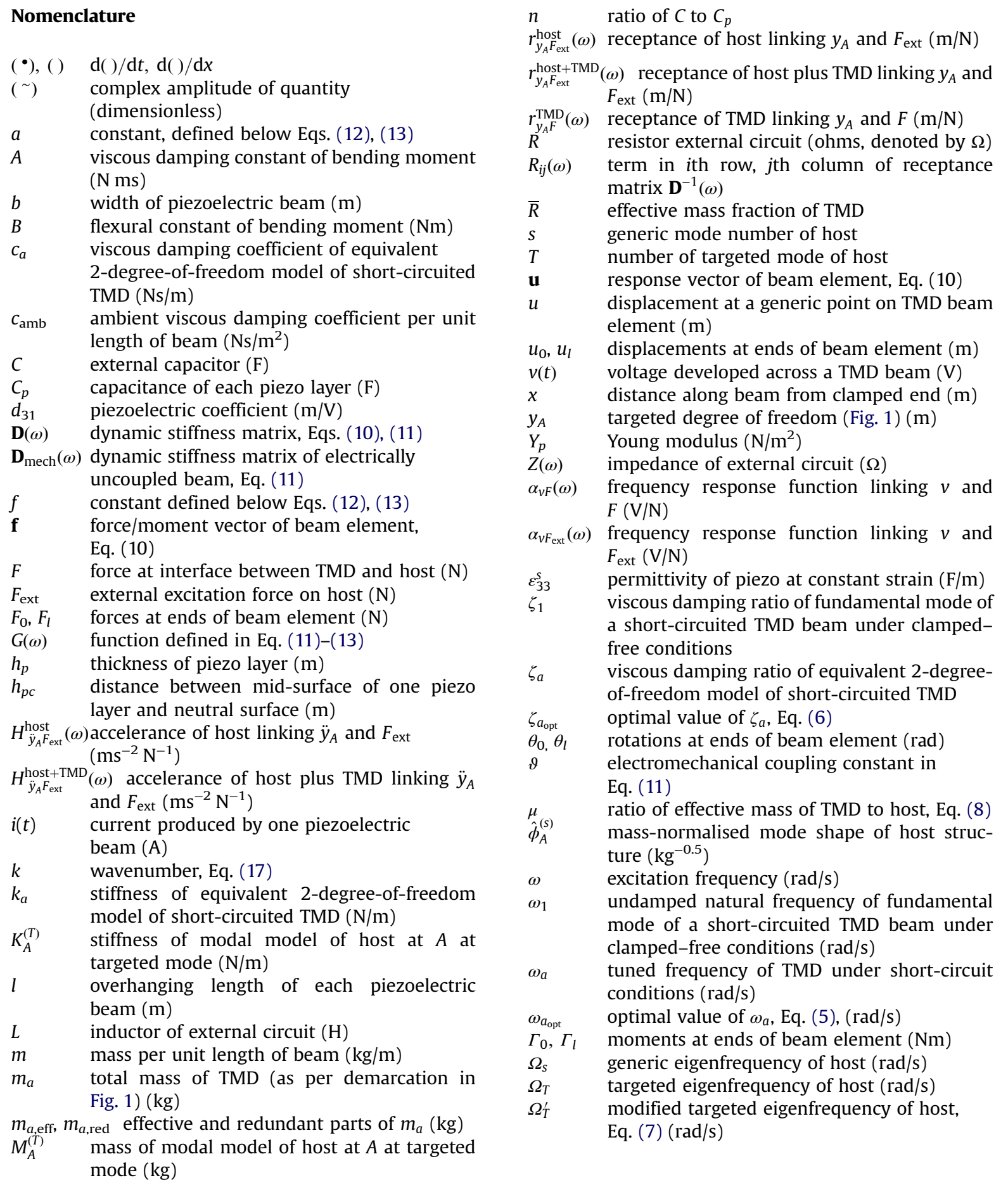

TMDs proposed and/or patented in [6,7] apply a constrained layer viscoelastic damping treatment to the beam, which has several disadvantages relative to the piezoelectric shunt damping method discussed in the next paragraph [5].

In the "electrical TVA" the auxiliary system is a piezoelectric patch directly bonded to the host structure and shunted by appropriate circuitry [8-13]. When shunted by an external inductor, resistor circuit and appropriately tuned, the patch is an electrical analogue of the TMD, targeting one mode [8-11]. The use of more complex shunt circuits and control strategies allows the targeting of more than one mode simultaneously by a single patch $[12,13]$. The electrical TVA is essentially an 


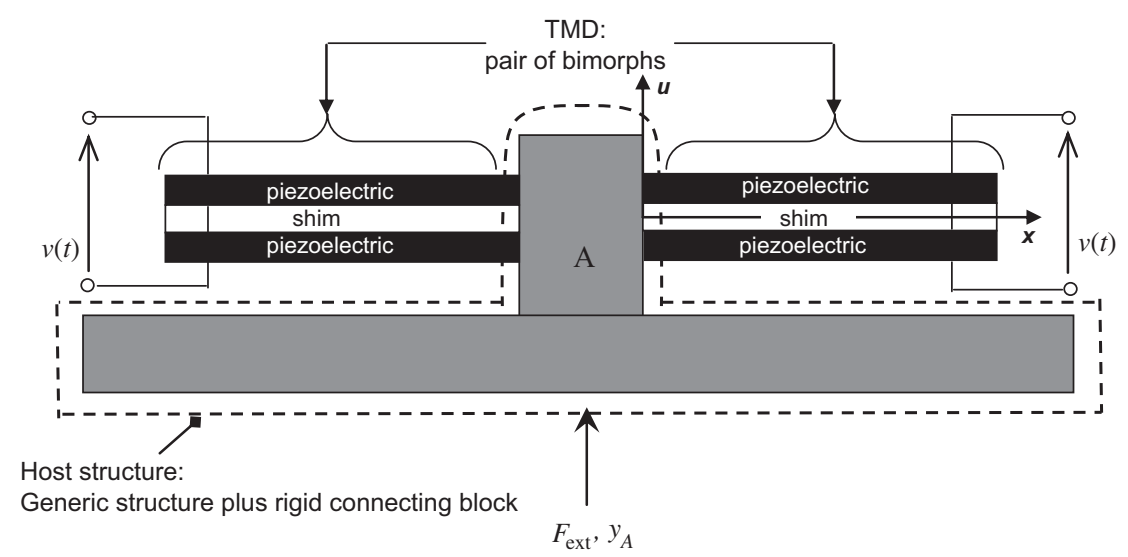

Fig. 1. Schematic of electromechanical TMD attached to a host structure [5].

advantageous alternative to conventional viscoelastic damping treatments [5,8-11]. However, when compared to the mechanical TMD, it is of limited application. Whereas the mechanical TMD can be used to suppress any vibration at a point, regardless of its cause, the piezoelectric patch is only effective against vibration arising from flexure of the host [5]. Moreover, whereas the mechanical TMD is readily applicable to any arbitrary host structure (the only data required are the frequency and mass of the targeted mode $[1,4,5])$, the analysis required to optimise the electrical TVA is not tractable to complex generic host structures [5].

A recent paper by the authors [5] introduced the novel concept of utilising a piezoelectric vibration energy harvesting (PVEH) beam as a TMD, thereby realising an "electromechanical" TMD. Such a TMD combines the relative advantages of the mechanical and (single-mode) electrical TVAs, apart from having the advantageous beam-like design. Fig. 1 shows the electromechanical TMD: it comprises a pair of piezoelectric beams (bimorphs) shunted by a resistor, capacitor and inductor connected in various alternative circuit configurations (the double-cantilever arrangement minimises rotational effects at the attachment point, as in practical realisations of beam-like TVAs [2,3]). The optimal damping required by this TMD is generated by the PVEH effect of the bimorphs. The concept was inspired by the largely overlooked fact that PVEH beams are, in many practical applications, mechanical absorbers of the vibration of the structure to which they are attached, as observed by the authors in [14]. The paper in [5] presented the theoretical basis of this dual PVEH/TMD beam device and verified the theory by two independent analytical methods [14]: the analytical modal analysis method and the dynamic stiffness method. The simulation results demonstrated that the benchmark degree of vibration attenuation, as defined by Den Hartog's classical theory [1,4], can be achieved through appropriate tuning of the circuitry for a device whose effective mass is less than 2 percent of the equivalent modal mass of the host structure.

The purpose of the present paper is to experimentally validate the concept and theory of the electromechanical TMD. As in [5], the piezoelectric beams in Fig. 1 are either separately connected across identical impedances ("separate circuits" configuration) or both connected across the same impedance ("single circuit" configuration). In each case the impedance is either a parallel arrangement of a capacitor, inductor and resistor $(C\|L\| R)$, or a capacitor in parallel with a series combination of an inductor and resistor $(C \|(L-R))$.

For completeness, the theory developed in [5] is represented in a concise form using exclusively the dynamic stiffness method (DSM) for the electromechanical analysis. The theory is further developed to predict the frequency response function of the voltage generated by the TMD, which was not studied in [5], and which is also experimentally investigated in this paper.

It is noted that, although the electromechanical TMD performs simultaneously the two functions of TMD and PVEH, the latter function can only be of any practical use if there is a means of energy storage. However, this would require nonlinear AC-DC rectification devices [15] and the analysis of such systems is outside the scope of this paper.

It is also noted that a similar observation on the vibration absorbing effects of energy harvesting devices was made by another author in a recent paper [16]. In that paper, a vibration absorber composed of a corrugated sheet of piezoelectric material shunted by a pure resistance load and surmounted by a top plate was proposed and used to suppress flexural vibrations of a plate while simultaneously harvesting energy. Unlike the research of the present paper, the optimisation of that device was not done with reference to a benchmark. Moreover, just like in the present paper, the electricity generated ("harvested") was not stored.

The following section (Section 2) presents the theory. The experimental design and procedure is described in Section 3. The experimental and theoretical results are presented and discussed in Section 4.

\section{Theory}

The purpose of a TMD is to dampen the targeted resonance peak of the frequency response function (FRF or receptance) connecting the response at the attachment point $y_{A}$ to the external excitation $F_{\text {ext }}$ on the host structure i.e. 
$\left|r_{y_{A} F_{\text {ext }}}^{\text {host }+ \text { TMD }}(\omega)\right| \ll\left|r_{y_{A} F_{\text {ext }}}^{\text {host }}(\omega)\right|$ for the range of excitation frequencies $\omega$ over which the targeted host structure mode is dominant, where $r_{y_{A} F_{\text {ext }}}^{\text {host }}(\omega), r_{y_{A} F_{\text {ext }}}^{\text {host }}(\omega)$ are defined as the complex ratio $\tilde{y}_{A} / \tilde{F}_{\text {ext }}$, with and without the TMD attached respectively, for harmonic functions $y_{A}=\operatorname{Re}\left\{\tilde{y}_{A} \mathrm{e}^{\mathrm{j} \omega t}\right\}, F_{\text {ext }}=\operatorname{Re}\left\{\tilde{F}_{\text {ext }} \mathrm{e}^{\mathrm{j} \omega t}\right\}$ (similar notation will be used throughout for other time-varying quantities). Fig. 1 illustrates the boundaries of the host structure and the TMD. Neglecting the damping in the original host structure, its receptance is given by [17]

$$
r_{y_{A} F_{\text {ext }}}^{\text {host }}(\omega)=\sum_{s=1}^{\infty} \frac{\left\{\hat{\phi}_{A}^{(s)}\right\}^{2}}{\Omega_{s}^{2}-\omega^{2}}
$$

where $\Omega_{S}$ is the circular frequency of the sth mode and $\hat{\phi}_{A}^{(s)}$ the corresponding mass-normalised mode-shape at the degree of freedom being targeted (the vertical displacement at $A$ in Fig. 1). It is noted from host/TMD demarcation in Fig. 1 that the modal parameters in Eq. (1) should include the effect of the attachment block and that part of the bimorphs is held inside the block. The mode to be targeted by the TMD is denoted as the Tth mode $(s=T)$, of frequency $\Omega_{T}$. Without loss of generality, taking $F_{\text {ext }}$ to be located at the targeted degree of freedom, it can be shown that the point receptance of the modified structure is given by

$$
r_{y_{A} F_{\text {ext }}}^{\text {host+TMD }}(\omega)=\frac{r_{y_{A} F_{\text {ext }}}^{\text {host }}(\omega)}{1+r_{y_{A} F_{\text {ext }}}^{\text {host }}(\omega) / r_{y_{A} F}^{\mathrm{TMD}}(\omega)}
$$

where $r_{y_{A} F}^{\mathrm{TMD}}(\omega)=\tilde{y}_{A} / \tilde{F}$, is the attachment point receptance of the TMD and $F$ is the force at the interface between the TMD and the host structure. The tuned frequency $\omega_{a}$ of the TMD is defined as its lowest undamped natural frequency with its base (point of attachment to the host structure) fixed; this frequency coincides with the lowest anti-resonance in $r_{y_{A} F}^{\mathrm{TMD}}(\omega)$ (under undamped conditions) [2,5]. When $\omega_{a}$ is roughly in the region of $\Omega_{T}$, the effect of the inclusion of the TMD, in the absence of any damping in it, is to split the original resonance peak at $\omega=\Omega_{T}$ in $r_{y_{A} F_{\text {ext }}}^{\text {host }}(\omega)$ into two peaks separated by an anti-resonance at $\omega=\omega_{a}$. With reference to Eq. (2), an optimised adjustment of the TMD's electrical parameters (and hence $\left.r_{y_{A} F}^{\mathrm{TMD}}(\omega)\right)$ then allows the replacement of the twin peaks and anti-resonance in $r_{y_{A} F_{\text {ext }}}^{\text {host }} \mathrm{TDD}_{(\omega)}(\omega y$ b plateau, thereby achieving the desired suppression. Such an optimisation requires a benchmark (target). The establishment of the benchmark is outlined in Section 2.1. The subsequent Section 2.2 will outline the derivation of $r_{y_{A} F}^{\mathrm{TMD}}(\omega)$ for given $R, L, C$ circuitry, with a view to optimise $r_{y_{A} F_{\text {ext }}}^{\text {host }}(\omega)$ via the adjustment of the electrical parameters. Section 2.3 derives an expression for the voltage developed by the TMD. The analysis assumes that the TMD beams are symmetrically disposed about the clamp and that rotational effects at the clamp are negligible. As in previous energy harvesting works e.g. [14,18,19], it is assumed that the electrodes bracketing the upper and lower surfaces of each piezoelectric layer of either beam (Fig. 1) are infinitely thin, flexible, have negligible resistance and run along the whole width $b$ and overhanging length $l$ of the beam.

\subsection{Establishing the benchmark}

The benchmark performance for the electromechanical TMD is derived by applying Den Hartog's classical TMD theory [4] to the proposed TMD in Fig. 1 under purely mechanical (electrically uncoupled) conditions (achieved by short-circuiting the bimorphs). This derivation requires two steps [5]: (a) reduction of the distributed-parameter beam structure of the TMD into an equivalent two-degree-of-freedom (2-dof) model; and (b) adaptation of Den Hartog's analysis [4] to the system formed by the combination of the 2-dof TMD model and the targeted modal component of the host structure. The parameters of the equivalent 2-dof model of the short-circuited electromechanical TMD are

$$
k_{a}=m_{a, \mathrm{eff}} \omega_{a}^{2}, \quad c_{a}=2 \zeta_{a} m_{a, \mathrm{eff}} \omega_{a}, \quad m_{a, \mathrm{eff}}=\bar{R} m_{a}, \quad m_{a, \text { red }}=(1-\bar{R}) m_{a}
$$

$m_{a}$ is the total mass of the TMD (comprising the two overhanging portions of the bimorphs) and $m_{a \text {,eff }}, m_{a, \text { red }}$ are the effective and redundant mass portions respectively, $\bar{R}$ being the effective mass fraction (for the present case of beams without a tip mass, $\bar{R}=60.49 \%$ [2]). In accordance with the definition of the tuned frequency $\omega_{a}$ given in the previous section, the tuned frequency $\omega_{a}$ and the viscous damping ratio $\zeta_{a}$ of the TMD bimorph absorber under short-circuit conditions are

$$
\omega_{a} \equiv \omega_{1}, \quad \zeta_{a} \equiv \zeta_{1}
$$

where $\omega_{1}, \zeta_{1}$ denote the undamped natural frequency and damping ratio of the fundamental mode of free vibration of either of the actual (distributed-parameter) electrically uncoupled beams with their base fixed in both translation and rotation ("clamped-free"). It is noted that the symbols $\omega_{a}$ and $\zeta_{a}$ are reserved for short-circuit conditions only (as observed in [14], the PVEH effect considered in the subsequent sections causes a slight shift in the tuned frequency and is typically not at all equivalent to an added viscous damper).

From Eq. (1), for excitation frequencies $\omega \approx \Omega_{T}$, the original host structure can approximately be represented as a massspring system of mass $M_{A}^{(T)}=1 /\left\{\hat{\phi}_{A}^{(T)}\right\}^{2}$ and stiffness $K_{A}^{(T)}=\Omega_{T}^{2} M_{A}^{(T)}$. The optimal tuned frequency $\omega_{a_{\mathrm{opt}}}$ and optimal viscous damping ratio $\zeta_{a_{\mathrm{opt}}}$ are then given by the following expressions [5]:

$$
\frac{\omega_{a_{\mathrm{opt}}}}{\Omega_{T}^{\prime}}=\frac{1}{1+\mu}
$$




$$
\zeta_{a_{\mathrm{opt}}}=\sqrt{\frac{3 \mu}{8(1+\mu)^{3}}}
$$

where the modified targeted host mode frequency $\Omega_{T}^{\prime}$ and mass ratio $\mu$ are given by

$$
\begin{gathered}
\Omega_{T}^{\prime}=\Omega_{T} \sqrt{M_{A}^{(T)} /\left\{M_{A}^{(T)}+m_{a, \text { red }}\right\}} \\
\mu=m_{a, \text { eff }} /\left\{M_{A}^{(T)}+m_{a, \text { red }}\right\}
\end{gathered}
$$

The attachment point receptance of the equivalent 2-dof TMD model is given by

$$
\left\{r_{y_{A} F}^{\mathrm{TMD}}(\omega)\right\}_{\text {uncoup }}^{2-\text { dof }}=\frac{\tilde{y}_{A}}{\tilde{F}}=\frac{-m_{a \text { eff }} \omega^{2}+k_{a}+\mathrm{j} \omega c_{a}}{-m_{a, \text { eff }} \omega^{2}\left(k_{a}+\mathrm{j} \omega c_{a}\right)-m_{a, \text { red }} \omega^{2}\left(-m_{a, \text { eff }} \omega^{2}+k_{a}+\mathrm{j} \omega c_{a}\right)}
$$

The benchmark performance of the TMD is defined as the response $r_{y_{\nu} F_{\text {ext }}}^{\text {hot }}$ TMD $(\omega)$ calculated from Eq. (2) with $\left\{r_{y_{A} F}^{\mathrm{TMD}}(\omega)\right\}_{\text {uncoup,opt }}^{2-\text { dof }}$ substituted for $r_{y_{A} F}^{\mathrm{TMD}}(\omega)$, where $\left\{r_{y_{A} F}^{\mathrm{TMD}}(\omega)\right\}_{\text {uncoup,opt }}^{2 \text {-dof }}$ is calculated from Eq. (9) with optimal values for $k_{a}$ and $c_{a}$ that are calculated from Eqs. (3) with $\omega_{a}$ and $\zeta_{a}$ set to their optimal values (defined by Eqs. (5) and (6)).

In practice, for the actual (distributed-parameter) TMD, it is easy to make $\omega_{1}\left(\equiv \omega_{a}\right.$, see Eq. (4a)) equal to $\omega_{a}$ opt. However, $\zeta_{a \text { opt }}$ is difficult to implement in practice. The difference between $\zeta_{a \text { opt }}$ and the actual value of $\zeta_{1}\left(\equiv \zeta_{a}\right.$, see Eq. (4b)) is addressed by the electrical tuning of the proposed TMD.

\subsection{Derivation of $\boldsymbol{r}_{\boldsymbol{y}_{\boldsymbol{A}} \boldsymbol{F}}^{\mathrm{TMD}}(\boldsymbol{\omega})$ for the electromechanical TMD [5]}

Let $\left(u_{0}, \theta_{0}\right),\left(u_{l}, \theta_{l}\right)$ denote the absolute displacement and rotation at the respective ends of either overhanging piezoelectric beam comprising the TMD. Let $\left(F_{0}, \Gamma_{0}\right),\left(F_{l}, \Gamma_{l}\right)$ denote the applied force and moment at these locations. Assuming harmonic vibration at circular frequency $\omega$ i.e. $u_{0}=\operatorname{Re}\left\{\tilde{u}_{0} \mathrm{e}^{\mathrm{j} \omega t}\right\}$, etc., the dynamic stiffness matrix $\mathbf{D}(\omega)$ of the piezoelectric beam relates the complex amplitudes of the force/moment excitation at its ends with the complex amplitudes of the corresponding displacements/rotations:

$$
\mathbf{f}=\mathbf{D}(\omega) \mathbf{u}, \quad \mathbf{f}=\left[\begin{array}{llll}
\tilde{F}_{0} & \tilde{\Gamma}_{0} & \tilde{F}_{l} & \tilde{\Gamma}_{l}
\end{array}\right]^{\mathrm{T}}, \quad \mathbf{u}=\left[\begin{array}{llll}
\tilde{u}_{0} & \tilde{\theta}_{0} & \tilde{u}_{l} & \tilde{\theta}_{l}
\end{array}\right]^{\mathrm{T}}
$$

The dynamic stiffness matrix can be expressed as [14]

$$
\mathbf{D}(\omega) \equiv \mathbf{D}_{\text {mech }}(\omega)+\vartheta G(\omega)\left[\begin{array}{cccc}
0 & 0 & 0 & 0 \\
0 & 1 & 0 & -1 \\
0 & 0 & 0 & 0 \\
0 & -1 & 0 & 1
\end{array}\right]
$$

The second matrix on the right hand side is the contribution from the PVEH effect. $\vartheta$ is the electromechanical coupling constant of the piezoelectric beam [14] and $G(\omega)$ is dependent, among other things, on the impedance of the external circuit. The origin of $G(\omega)$ is explained by consideration of the current generated by one piezoelectric beam, which is obtained by integrating the charge density (defined by the piezoelectric constitutive relation [14]) over the electrode area and differentiating with respect to time. For a piezoelectric beam that is individually connected to an (external) impedance $Z(\omega)$, as in the separate circuits configuration [5]:

$$
\begin{gathered}
\tilde{v}=G\left(\tilde{\theta}_{l}-\tilde{\theta}_{0}\right) \\
G(\omega)=\frac{-\mathrm{j} \omega f d_{31} Y_{p} h_{p c} b}{\mathrm{j} \omega(f / a) C_{p}+1 / Z(\omega)}
\end{gathered}
$$

In the above equations: $v$ is the output voltage developed across the piezoelectric beams (see Fig. 1 ); $d_{31}$ is the piezoelectric coefficient, $Y_{p}$ is the Young modulus of the piezoelectric material; $h_{p c}$ is the distance between the mid-surface of one piezo layer and the neutral surface of the piezoelectric beam's composite cross-section; $f=1$ and $a=2$ for a bimorph with the upper and lower piezo layers wired in series (as in the present case, Fig. 1) [14]; $C_{p}$ is the capacitance of each piezo layer of dimensions $l \times b \times h_{p}$, given by

$$
C_{p}=\frac{\varepsilon_{33}^{s} b l}{h_{p}}
$$

$\varepsilon_{33}^{s}$ being the permittivity at constant strain.

Substituting the relevant expression for $G(\omega)$ into Eq. (13) and defining $n=C / C_{p}$, the following expressions are obtained for the separate circuits configuration:

$$
G(\omega)=\frac{\omega^{2} f d_{31} Y_{p} h_{p c} b}{1 / L+\mathrm{j} \omega / R-\omega^{2} C_{p}(f / a+n)} \quad \text { for } C\|L\| R \text { impedance }
$$




$$
G(\omega)=\frac{-f d_{31} Y_{p} h_{p c} b\left(\mathrm{j} \omega R-\omega^{2} L\right)}{C_{p}\left\{1 / C_{p}+(\mathrm{j} \omega / R)(f / a+n)-\omega^{2} L(f / a+n)\right\}} \quad \text { for } C \|(L-R) \text { impedance }
$$

For the single circuit configuration, the currents generated by each bimorph are added together before delivery to the external circuit; hence, assuming a perfect mechanical and electrical symmetry, the expressions for $G(\omega)$ are obtained by replacing $f$ by $2 f$ in Eqs. (15), (16) [5].

The first term of Eq. (11) is the dynamic stiffness matrix of the beam under electrically uncoupled conditions. Its elements are given in [14] and are expressions of the wavenumber $k$ :

$$
\begin{gathered}
k=\omega^{1 / 2}\left\{\frac{m}{\hat{B} /\left[1-\mathrm{j} c_{\mathrm{amb}} /(m \omega)\right]}\right\}^{1 / 4} \\
\hat{B}=B[1+\mathrm{j} \omega A / B]
\end{gathered}
$$

where $m$ is the mass per unit length of the beam, $c_{\mathrm{amb}}$ is the ambient damping coefficient per unit length, and $B, A$ are respectively the bending stiffness and bending damping constants of the composite cross-section of the piezoelectric beam. If $c_{\mathrm{amb}}$ is neglected, then $A$ can be calculated from an experimentally determined value of $\zeta_{1}$ [14]:

$$
A=\frac{2 B}{\omega_{1}} \zeta_{1}
$$

It is shown in [14] that determination of $A$ by this method introduces no error in the FRF level of the beam at its fundamental clamped-free mode. The method is hence justified in the present application since, by definition (Eqs. (4a,b)), the bimorph TMD is designed to be effective around its first clamped-free mode (regardless of the targeted mode of the host structure).

The expression for $r_{y_{A} F}^{\mathrm{TMD}}(\omega)$ is obtained by solving Eq. (10a) for $\mathbf{u}$, then setting $\tilde{\theta}_{0}=0$, in addition to $\tilde{F}_{l}, \tilde{\Gamma}_{l}=0$, expressing $\tilde{\Gamma}_{0}$ in terms of $\tilde{F}_{0}$ and substituting $\tilde{u}_{0}=\tilde{y}_{A}$ and $\tilde{F}_{0}=\tilde{F} / 2$ (in order to account for the two symmetric bimorphs constituting the TMD):

$$
r_{y_{A} F}^{\mathrm{TMD}}(\omega)=\frac{\tilde{y}_{A}}{\tilde{F}}=\frac{1}{2}\left\{R_{11}(\omega)-R_{12}(\omega) R_{21}(\omega) / R_{22}(\omega)\right\}
$$

where $R_{i j}(\omega)$ is the term in $i$ th row, $j$ th column of the receptance matrix $\mathbf{D}^{-1}(\omega)$. This expression is then used within Eq. (2) to obtain $r_{y_{A} F}^{\text {host }+\mathrm{TMD}}(\omega)$.

\subsection{Derivation of the voltage developed by the electromechanical TMD}

Let $\alpha_{v F}(\omega)$ denote the FRF relating the voltage $v$ with the interface force $F$ (voltage generated per unit interface force). From Eq. (12), noting that $\tilde{\theta}_{0}=0$ :

$$
\alpha_{v F}(\omega)=\frac{\tilde{v}}{\tilde{F}}=G \frac{\tilde{\theta}_{l}}{\tilde{F}}
$$

The expression for $\tilde{\theta}_{l}$ is obtained by solving Eq. (10a) for $\mathbf{u}$, then setting $\tilde{\theta}_{0}=0$, in addition to $\tilde{F}_{l}, \tilde{\Gamma}_{l}=0$, expressing $\tilde{\Gamma}_{0}$ in terms of $\tilde{F}_{0}$ and substituting $\tilde{F}_{0}=\tilde{F} / 2$. The expression for $\alpha_{v F}(\omega)$ in Eq. (21) then becomes:

$$
\alpha_{v F}(\omega)=\frac{1}{2} G(\omega)\left\{R_{41}(\omega)-R_{42}(\omega) R_{21}(\omega) / R_{22}(\omega)\right\}
$$

With reference to Fig. 1, what is actually of interest is the FRF relating the voltage $v$ with the external force $F_{\text {ext }}$ (voltage generated per unit external force), denoted by $\alpha_{v F_{e x t}}(\omega)$. This FRF is obtained as follows:

$$
\alpha_{v F_{\mathrm{ext}}}(\omega)=\frac{\tilde{v}}{\tilde{F}_{\mathrm{ext}}}=\left(\frac{\tilde{y}_{A} / \tilde{F}_{\mathrm{ext}}}{\tilde{y}_{A} / \tilde{F}}\right) \frac{\tilde{v}}{\tilde{F}}=\frac{r_{y_{A} F_{\mathrm{ext}}}^{\mathrm{host}+\mathrm{TMD}}(\omega)}{r_{y_{A} F}^{\mathrm{TMD}}(\omega)} \alpha_{v F}(\omega)
$$

\section{Experimental design and procedure}

Fig. 2(a) and (b) respectively show photographs of the experimental set-ups for the separate circuits and single circuit configurations of the electromechanical TMD. The electromechanical TMD, composed of two symmetrical shunted bimorph beams, was attached to the host structure via the attachment block. The host structure was mounted on an electrodynamic shaker which provided the external excitation ( $F_{\text {ext }}$ in Fig. 1$)$. The theory developed in the previous section applied for a generic host structure. However, without loss of generality, the host structure in the experimental validation study was taken to be a free-free beam with an attachment block in the middle and the TMD was targeted at dampening its first flexural mode. It is noted that, like the classical TMD, the proposed TMD acts directly on only one degree of freedom at its point of attachment. Hence, if it was desired to target the second flexural mode of the free-free beam, the TMD would need to be attached at a different point along the beam since the beam's midpoint is a node for displacement in that mode 
(a)

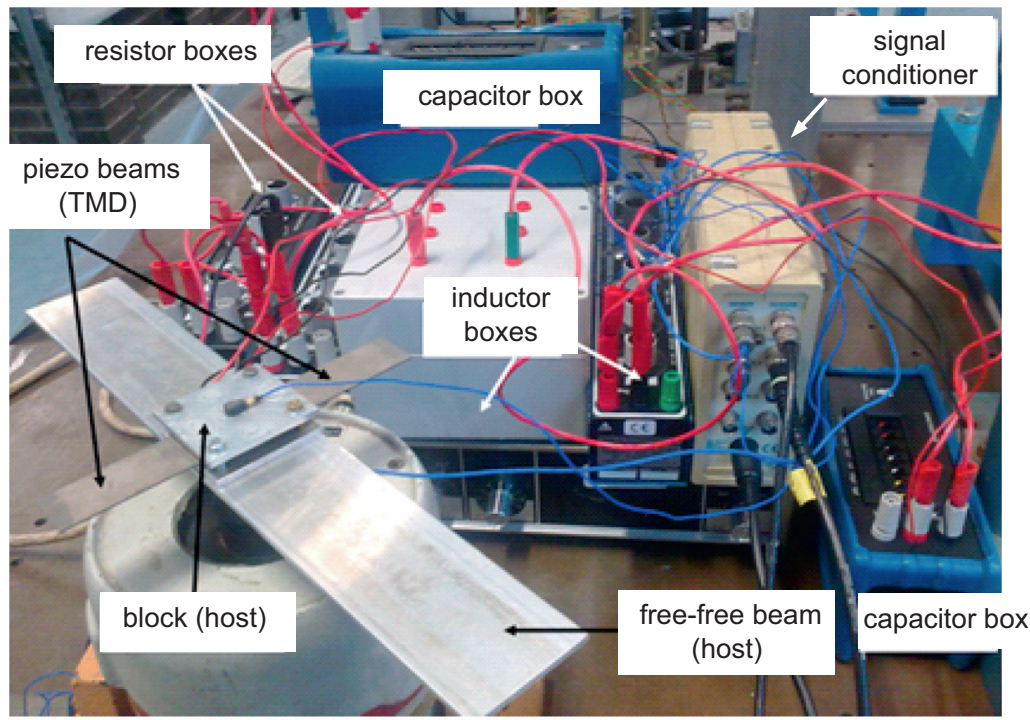

(b)

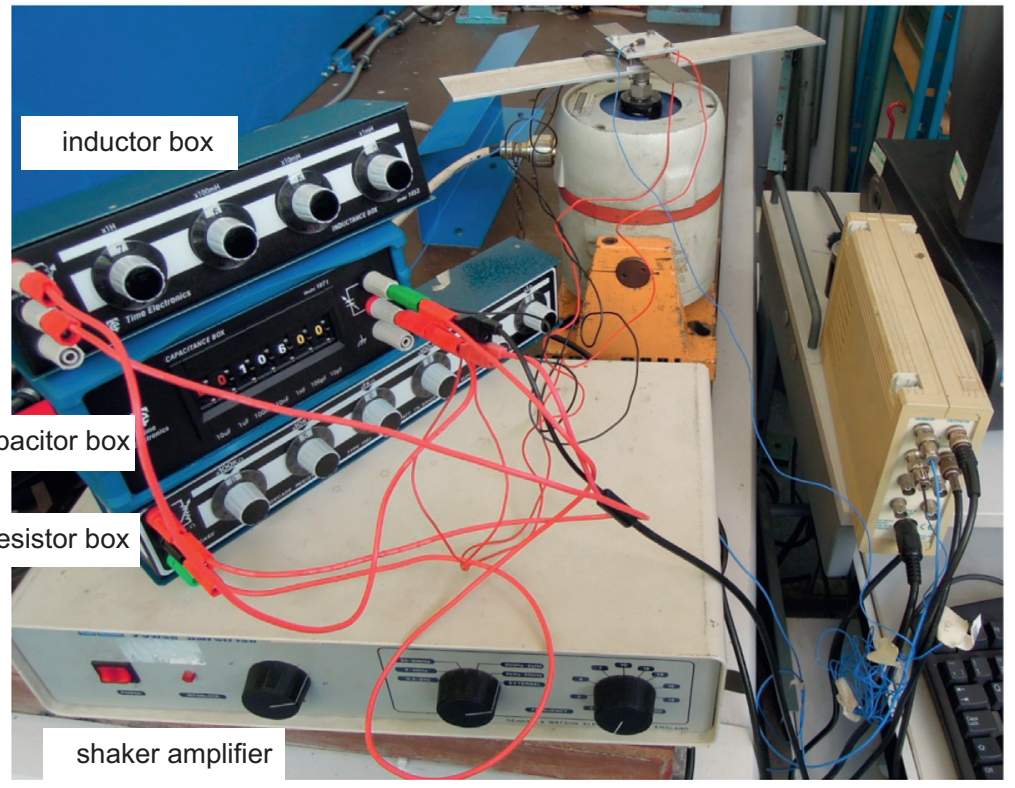

Fig. 2. Experimental set-up for testing electromechanical TMD performance: (a) separate circuits configuration and (b) single circuit configuration.

(a convenient location in that case would be around a fifth of the total length away from the middle since the displacement there would be substantial, with minimal slope rotation).

The main design and development activities performed in order to produce the experimental set-up can be summarised as follows:

- Mechanical aspect:

- Experimental identification of the modal parameters (resonance frequency $\Omega_{T}$, equivalent mass $M_{A}^{(T)}$ ) of the targeted mode of the host structure.

- Design and procurement of the necessary bimorph beams that, when short circuited, had a fundamental clampedfree frequency $\omega_{a}$ (Eq. (4a)) that satisfied the TMD optimal tuning condition defined by Eq. (5), for a given prescribed mass ratio (defined by Eq. (8)).

- Electrical aspect:

$\circ$ Design and development/procurement of $R, C, L$ circuitry that could be easily adapted to any one of the four configurations considered $(C\|L\| R$ or $C \|(L-R)$ impedance in either a separate circuits or single circuit configuration, see Introduction), based on calculations that optimised the circuit parameters against the benchmark established in Section 2.1 . 


\subsection{Experimental design-mechanical aspect}

The host structure (i.e. free-free beam with attachment block, without TMD, Fig. 2(a)) was mounted on the shaker via a force gauge which measured $F_{\text {ext }}(t)$. The signal $\ddot{y}_{A}(t)$ was measured by a tiny accelerometer and conditioned prior to being sent to the data acquisition system. A pc-controlled data-acquisition system was used to generate the excitation signal (sent to the shaker via an amplifier) and to acquire the signals $\ddot{y}_{A}(t), F_{\text {ext }}(t)$. Random excitation (band-limited white noise) was fed to the shaker and the FRF $H_{\dot{y}_{A} F_{\text {ext }}}^{\text {host }}(\omega)\left\{=-\omega^{2} r_{y_{A} F_{\text {ext }}}^{\text {host }}(\omega)\right\}$ was calculated by the data acquisition system software from the acquired signals.

The undamped natural frequency and damping ratio of the targeted mode were determined by applying the circle-fit method [17] to the measured FRF. A multimodal curve fitting method [17] was then used to determine the mass-normalised mode shapes from the measured FRF. This method was based on the modal series expansion of Eq. (1), which neglects damping in the structure. The series expansion was truncated beyond the second mode (which is the first flexural mode, the first mode simply defining rigid body motion (i.e. $\Omega_{1}=0, \Omega_{2}=\Omega_{T}$ )). The measured FRF was then checked against the one reconstructed from Eq. (1) using the identified modal parameters. In this reconstruction, a damping term of $\mathrm{j} \hat{\zeta}_{2} \Omega_{2} \omega$ was added to the denominator of the second term of the series expansion of Eq. (1) (where the host structure damping $\hat{\zeta}_{2}=0.14 \%$ ). Agreement in resonance amplitude level in the flexural mode between the measured and reconstructed FRFs confirmed that the damping present in the host structure was indeed negligible.

According to Fig. 1, the TMD, of mass $m_{a}$, is comprised of the overhanging portions of the bimorphs. The mass of the overhanging part of each of the required bimorph beams is $m_{a} / 2$, and their first clamped-free natural frequency under short circuited conditions is the tuned frequency of the TMD $\omega_{a}$ (as per Eq. (4a)). Having determined $M_{A}^{(T)}$ and prescribing a mass ratio $\mu$ in the region of 2 percent, the required value of $m_{a}$, was estimated from Eq. (8) and (3c,d). The required tuned frequency $\omega_{a}$ was then estimated from Eqs. (5) and (7) such that it was optimal. The bimorphs were then specified giving due consideration to Eq. (4a) where [18]:

$$
\omega_{1}=(1.87510 / l)^{2} \sqrt{B / m}
$$

The above described calculations determined the choice of the bimorphs, which were manufactured by Piezo Systems Inc. Each comprised two PZT-5A4E layers bonded to the top and bottom surfaces of an aluminium shim. Each bimorph had series-connected layers and its geometric, material and electromechanical properties, as provided by the manufacturer, are given in Table 1(a). It is noted that the total length of the bimorph as received from the manufacturer was 72.5 mm. Of this length, $58.75 \mathrm{~mm}$ was overhung to attain the required tuned frequency. The clamped length of the piezos could be regarded as a simple rigid mass addition to the host structure. Hence, with the TMD attached, the host structure parameters $M_{A}^{(1)}$, $M_{A}^{(2)}\left(=M_{A}^{(T)}\right), \Omega_{2}\left(=\Omega_{T}\right.$ ) were very slightly corrected to account for this, their final values being given in Table 1(b).

Using the relevant parameters in Table 1, the final value of the mass ratio recalculated from Eq. (8) and (3c,d) was $\mu=1.93 \%$. Also, from Eqs. (4a), (24), (5) and (7) it was evident that $\omega_{1}$ (i.e. $\left.\omega_{a}\right) \approx \omega_{a_{\text {opt }}}$ i.e. the electrically uncoupled system was approximately optimally tuned. Using Eq. (6), the optimal damping ratio was calculated to be $\zeta_{a_{\mathrm{opt}}}=8.28 \%$. This value was used for the benchmark model, against which the experimental and theoretical performances of the electromechanical TMD were judged.

\subsection{Experimental design-electrical aspect}

The theoretical simulations performed in [5], covering a broad range of performance, including the optimal one for each circuit configuration in Figure, considered $R, L, C$ parameters in the following ranges:

- Inductors $L$ in the range $7.66-13.5 \mathrm{H}$.

- Resistances $R$ in the range of $100-1000 \mathrm{k} \Omega$.

Table 1

Parameters of electromechanical TMD (a) and modal parameters of host structure (b).

(a) Parameters of electromechanical TMD

\begin{tabular}{|c|c|c|}
\hline \multicolumn{2}{|c|}{ Overhanging length of each beam, $l(\mathrm{~mm})$} & 58.75 \\
\hline \multicolumn{2}{|c|}{ Width of each beam, $b(\mathrm{~mm})$} & 25 \\
\hline \multicolumn{2}{|c|}{ Thickness of each piezoelectric layer, $h_{p}(\mathrm{~mm})$} & 0.267 \\
\hline \multicolumn{2}{|c|}{ Thickness of each shim $h_{s h}(\mathrm{~mm})$} & 0.285 \\
\hline \multicolumn{2}{|c|}{ Young modulus of the piezoelectric, $Y_{p}(\mathrm{GPa})$} & 66 \\
\hline \multicolumn{2}{|c|}{ Young modulus of the shim, $Y_{s h}(\mathrm{GPa})$} & 72 \\
\hline \multicolumn{2}{|c|}{ Mass of TMD, $m_{a}$ (comprising overhanging portions of beams) $(\mathrm{kg})$} & $15 \times 10^{-3}$ \\
\hline \multicolumn{2}{|c|}{ piezoelectric coefficient $d_{31}(\mathrm{pm} / \mathrm{V})$} & \multirow{3}{*}{$1.3555 \times 10^{-8}$} \\
\hline permittivity a & $\operatorname{strain} \varepsilon_{33}^{s}(\mathrm{~F} / \mathrm{m})$ & \\
\hline \multicolumn{2}{|c|}{ (b) Modal parameters of host structure } & \\
\hline$\Omega_{1} /(2 \pi)(\mathrm{Hz})$ & $M_{A}^{(1)}=1 /\left\{\hat{\phi}_{A}^{(1)}\right\}^{2}(\mathrm{~kg})$ & 0.278 \\
\hline$\Omega_{2} /(2 \pi)(\mathrm{Hz})$ & $M_{A}^{(2)}=1 /\left\{\hat{\phi}_{A}^{(2)}\right\}^{2}(\mathrm{~kg})$ & 0.463 \\
\hline
\end{tabular}


Capacitors in the range $74-400 \mathrm{nF}$ (the capacitance $C_{p}$ of each piezo layer was calculated from Eq. (14) to be $74.565 \mathrm{nF}$, which closely matched with the measured value of $76 \mathrm{nF}$ ).

The resistances and capacitances required were available from off-the-shelf variable resistance and capacitance boxes (two of each, in the case of the separate circuit configuration). The inductor requirement was covered by using one off-theshelf variable inductor box, which provided inductances of up to $12.11 \mathrm{H}$ (with a resolution of $0.001 \mathrm{H}$ ), and a custom-made variable inductor box, which provided inductances in the range $0.5-20 \mathrm{H}$ (with a resolution of $0.5 \mathrm{H}$ ). This latter box contained five copper-wound inductors of different values that could be interconnected in different ways; this not only produced a range of values of inductance, but could also be used to augment the range of the other inductance box; hence, for example, it was possible to produce a separate circuits configuration with an inductance of $13 \mathrm{H}$ in each circuit.

\subsection{Experimental procedure}

The experimental validation procedure was similar to that described in Section 3.1, except that the shunted electromechanical TMD was attached to the host structure, as in Figs. 1 and 2, and the generated voltage $v(t)$ was acquired, in addition to $\ddot{y}_{A}(t), F_{\text {ext }}(t)$. The signal $v(t)$ was measured by the data acquisition system itself (i.e. connected directly to it from the circuit). Band-limited white noise was fed to the shaker and the FRFs $H_{\dot{y}_{A} F_{\text {ext }}}^{\text {hot }}$ TMD $(\omega)\left\{=-\omega^{2} r_{y_{A} F_{\text {ext }}}^{\text {host }+\operatorname{TMD}}(\omega)\right\}, \alpha_{v F_{\text {ext }}}(\omega)$ were calculated by the data acquisition software. Linearity was ascertained by monitoring the coherence function between the acquired signals, as in [18].

Prior to performing the tests presented in this paper, tests similar to those in $[18,19]$ were performed to determine the damping ratio of the fundamental clamped-free mode of the electrically uncoupled bimorphs ( $\zeta_{1}$, see Eq. (4b)), which was necessary for the determination of the bending damping constant $A$ (Eq. (19)). By applying the fitting techniques in [18] or [19] to the FRF relating $v(t)$ to $\ddot{y}_{A}(t), \zeta_{1}$ was estimated to be 1 percent. The value for $A$ used in all theoretical results for $r_{y_{A} F_{\text {ext }}}^{\text {host }}(\omega)$ in the subsequent section was based on this value of $\zeta_{1}$.

\section{Experimental and theoretical results}

Fig. 3 shows satisfactory agreement between the experimental and theoretical results for $r_{y_{A} F_{\text {ext }}}^{\text {host }}{ }^{2}(\omega)$ for the case where the bimorphs were short-circuited. The theoretical result was obtained using the dynamic stiffness equations in Section 2.2 by setting $G(\omega) \equiv 0$. Also shown in Fig. 3 are the benchmark response of the TMD and the experimental result for $r_{y_{A} F_{\text {ext }}}^{\text {host }}(\omega)$. The benchmark performance, denoted by $\left\{r_{y_{A} F_{\text {ext }}}^{\text {host } T M D}(\omega)\right\}_{\text {nncoupott }}$, was calculated using the equivalent two-degree-of-freedom model of the TMD, as per penultimate paragraph of Section 2.1, and so is based on $\zeta_{a}=\zeta_{a}$ opt $(=8.24$ percent). It is noted that a virtually identical benchmark performance was obtained by calculating $r_{y_{A} \mathrm{~F}_{\mathrm{ext}}}^{\text {host }}(\omega)$ using the dynamic stiffness equations in Section 2.2 (which refer to the actual distributed-parameter TMD) with $G(\omega) \equiv 0$ and a value for $A$ based on $\zeta_{1}$ set to $\zeta_{a \mathrm{opt}}(=8.24$ percent $)$ instead of the actual value of 1 percent.

In the subsequent sections, the electrical coupling (PVEH) effect is exploited to make up for the shortfall in the actual damping level relative to the optimal value. As stated in [5], previous research e.g. [14,18,19] has proved that the PVEH effect is typically not at all equivalent to an added viscous damper (unless the electrical load is a pure resistance of sufficiently low value [14]). However, the following experimental and theoretical results will show that this fact is no obstacle to achieving a desired response $r_{y_{A} F_{\text {ext }}}^{\text {host }}$ (MDD $(\omega)$ with the electromechanical TMD that is at least as good as that achieved by the benchmark TMD.

In the following sections, for each of the four circuit configurations considered, the experimental and theoretical results for $r_{y_{A} F_{\text {ext }}}^{\text {host }}(\omega)$ were both optimised with the aim of making $\left|r_{y_{A} F_{\text {ext }}}^{\text {host }+\mathrm{TMD}}(\omega)\right|$ less than or equal to the benchmark function $\left|\left\{r_{y_{A} F_{\text {ext }}}^{\text {host+TMD }}(\omega)\right\}_{\text {uncoup.opt }}\right|$ over a wide frequency range. In the case of the experimental results, this was done by manual tuning of the $R, L, C$ parameters, using the predicted (i.e. theoretical) optimal parameters as a guide. In the case of the theoretical results, the $R, L, C$ parameters were optimised using the Matlab Optimization Toolbox [20], following the

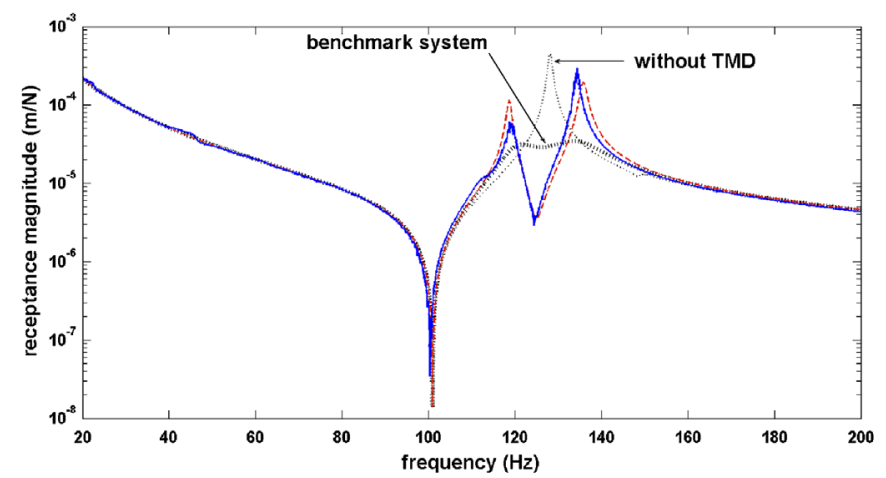

Fig. 3. Theoretical (unlabelled dashed line) and experimental (solid line) receptance of host structure with short-circuited TMD attached. 
procedure described in [5]. For a given external capacitance $C=n C_{p}$ the theoretical optimisation process used an initial approximation for $L$ that approximately maximised the electrical effect $G$ over the frequency range of the targeted mode (where $\omega \approx \omega_{a}$ ); for the separate circuits case, the denominator of Eqs. (15) or (16) is approximately minimised for

$$
L_{\mathrm{opt}, \mathrm{approx}}=\frac{1}{C_{p}(n+f / a) \omega_{a}^{2}}
$$

The above approximation applies for the separate circuit cases; for the single circuit configuration, the approximation is given by replacing replacing $f$ by $2 f$ in Eq. (25).
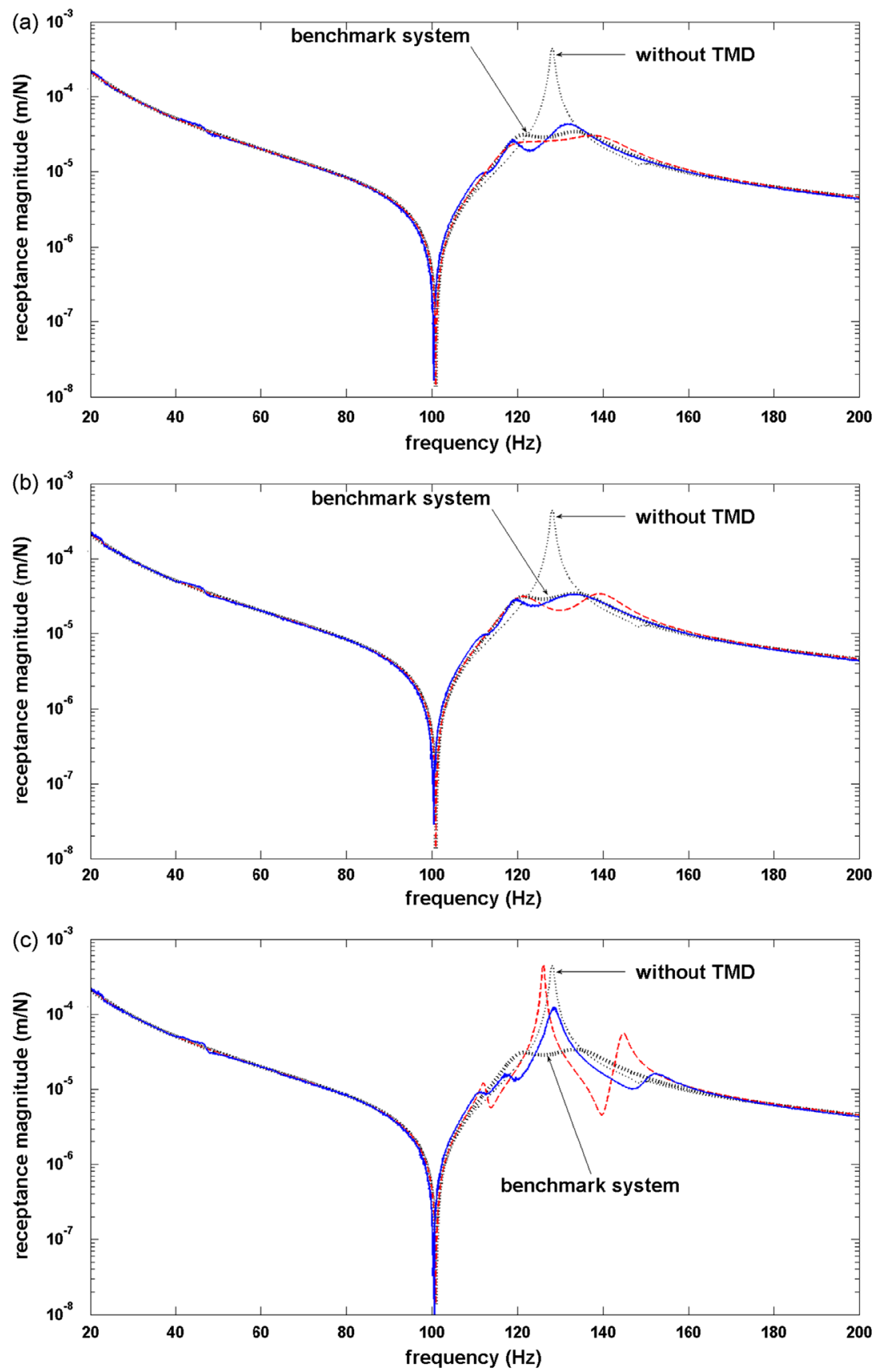

Fig. 4. Theoretical (unlabelled dashed line) and experimental (solid line) receptance of host structure with TMD attached, single circuit configuration with $C\|L\| R$ impedance, at three different conditions: (a) theoretical optimal conditions $R=22.5 \mathrm{k} \Omega, L=10 \mathrm{H}, C=1.05 C_{p}$; (b) experimental optimal conditions $R=22.5 \mathrm{k} \Omega, L=10.5 \mathrm{H}, C=1.18 C_{p}$; and (c) non-optimal conditions $R=500 \mathrm{k} \Omega, L=10 \mathrm{H}, C=1.05 C_{p}$. 


\subsection{Single circuit with $C\|L\| R$ load}

The optimal electrical parameters were predicted to be $R=22.5 \mathrm{k} \Omega, L=10 \mathrm{H}, C=1.05 C_{p}$ and Fig. 4(a) shows the predicted and measured FRFs $r_{y_{A} F_{\mathrm{ext}}}^{\text {host } \mathrm{TMD}}(\omega)$ for these parameters; the experimental response was near, but not quite, optimal. Fig. 4(b) shows that optimal conditions were achieved experimentally for slightly different parameters $(R=22.5 \mathrm{k} \Omega, L=10.5 \mathrm{H}$, $C=1.18 C_{p}$ ); this figure also contains the theoretical prediction for the same parameters. The results show that: (i) there was reasonable agreement between the predicted and measured FRFs for the same electrical parameters; (ii) a response that was at least as good as the benchmark was indeed achievable experimentally as well as theoretically; (iii) the experimental optimal response was achieved for electrical parameters that were approximately in the region of the corresponding predictions. Fig. 4(c) shows that the predicted and measured responses followed the same trend as the resistance was increased to a non-optimal value of $500 \mathrm{k} \Omega$.

Fig. 5(a-c) shows reasonable agreement between the predicted and measured voltage FRFs $\alpha_{v F_{\text {ext }}}(\omega)$ for electrical parameters respectively corresponding to those in Fig. 4(a-c). Fig. 5(a,b) shows that optimal conditions resulted in a broad peak in the voltage FRF over the region of the targeted mode; this plateau disappeared as the conditions were made significantly non-optimal (Fig. 5(c)).

It is noted that research into the electrical TVA (shunted piezoelectric patch) typically did not include an external capacitor e.g. [8]. The inclusion of the external capacitor $C$ in the proposed TMD is justified by two reasons: it reduces the
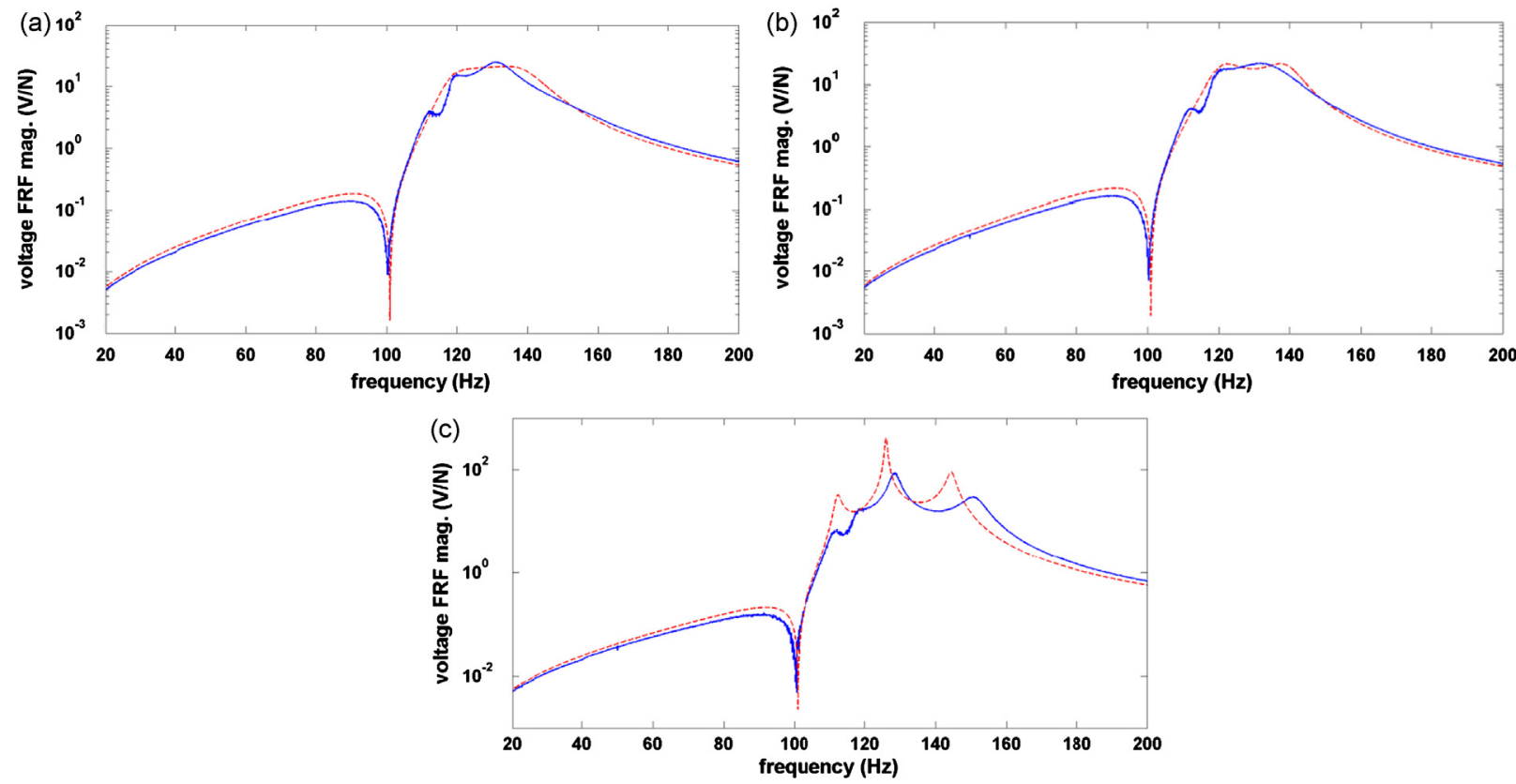

Fig. 5. Theoretical (dashed line) and experimental (solid line) voltage FRF (voltage $v$ per unit excitation force $F_{\text {ext }}$ ) for single circuit configuration with $C\|L\| R$ impedance. (a), (b) and (c) respectively correspond to the conditions in Fig. 4(a), (b) and (c).

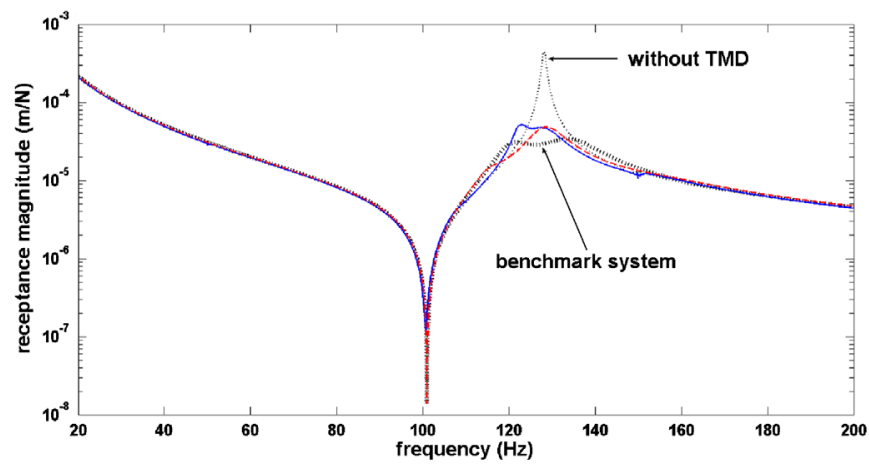

Fig. 6. Theoretical (unlabelled dashed line) and experimental (solid line) receptance of host structure with TMD attached, single circuit configuration with $L \| R$ impedance (no external capacitor) at experimental optimal conditions $R=40 \mathrm{k} \Omega, L=15.8 \mathrm{H}$. 
size of the optimised inductor (as indicated approximately by Eq. (25)); it introduces an additional controllable parameter that is usually important for achieving the targeted (benchmark) response. This is illustrated by the results presented in Fig. 6, in which the external capacitor $C$ was omitted: it is seen that the optimal (best possible) experimental response achieved was considerably poorer than the benchmark and the inductor necessary $(15.8 \mathrm{H})$ was significantly higher than that required if $C$ were included (10.5 H, Fig. 4(b)).

\subsection{Single circuit with $C \|(L-R)$ load}

The advantage of using $C \|(L-R)$ over $C\|L\| R$ was that the $L-R$ combination increased the net impedance of the system. Hence, as seen from Fig. 7(a,b), the $C \|(L-R)$ system attained tuned conditions at much lower values of $R$ than the $C\|L\| R$ system. Optimum
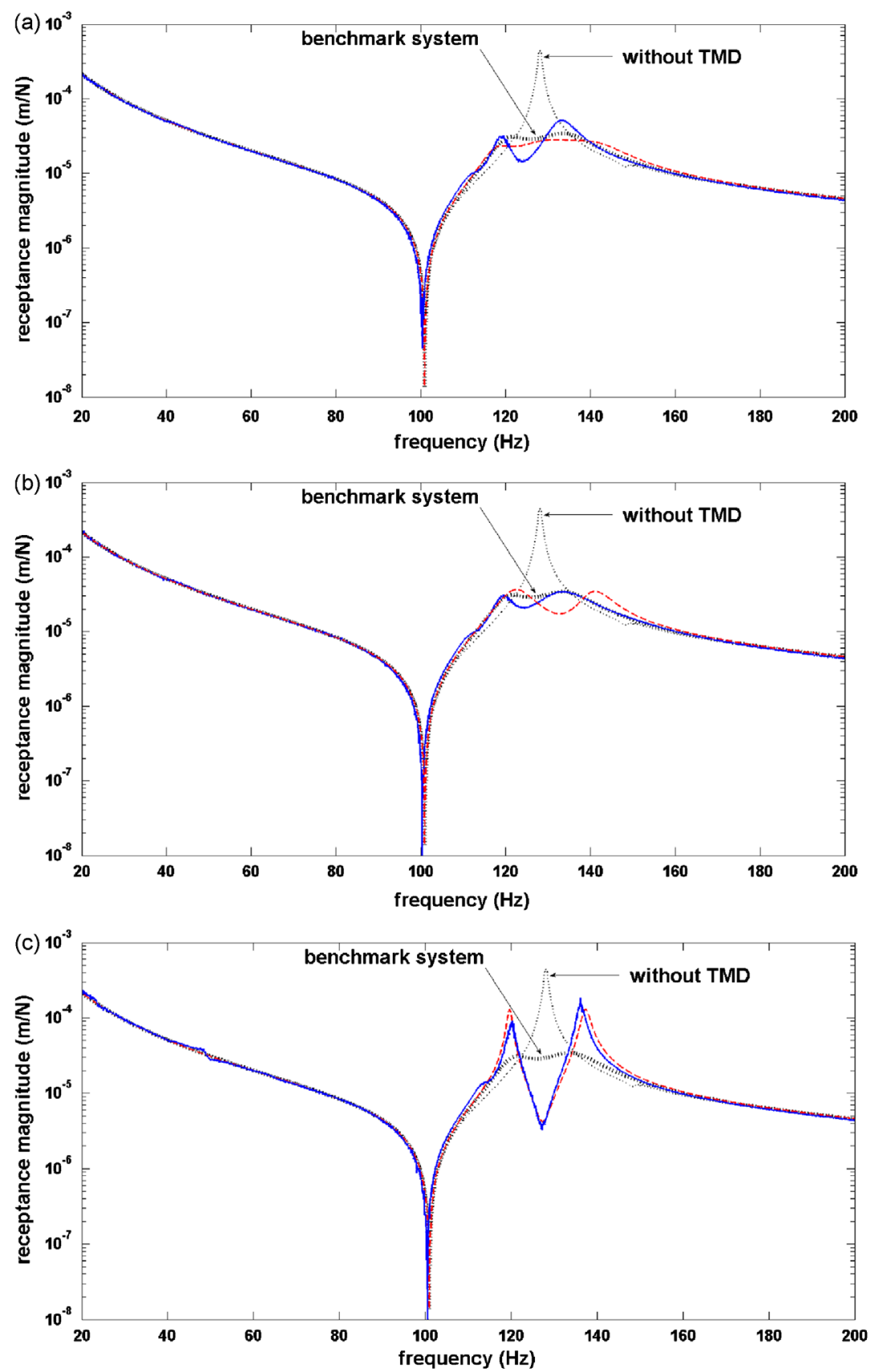

Fig. 7. Theoretical (unlabelled dashed line) and experimental (solid line) receptance of host structure with TMD attached, single circuit configuration with $C \|(L-R)$ impedance, at three different conditions: (a) theoretical optimal conditions $R=1.8 \mathrm{k} \Omega, L=7.66 \mathrm{H}, C=1.42 C_{p}$; (b) experimental optimal conditions $R=1.8 \mathrm{k} \Omega, L=9 \mathrm{H}, C=1.42 C_{p}$; and (c) non-optimal conditions $R=50 \mathrm{k} \Omega, L=7.66 \mathrm{H}, C=1.42 C_{\boldsymbol{p}}$. 

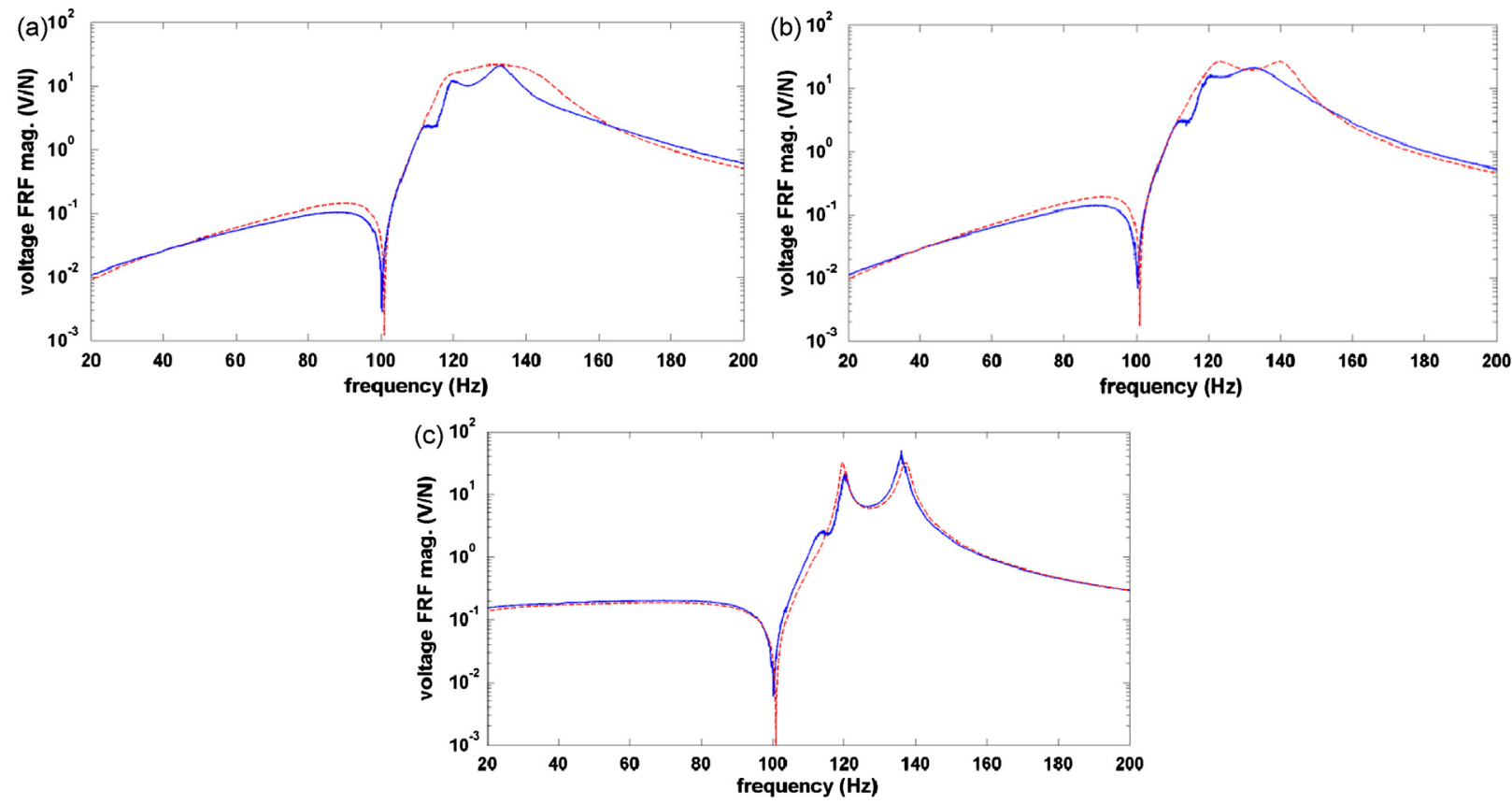

Fig. 8. Theoretical (dashed line) and experimental (solid line) voltage FRF (voltage $v$ per unit excitation force $F_{\text {ext }}$ ) for single circuit configuration with $C \|(L-R)$ impedance. (a), (b) and (c) respectively correspond to the conditions in Fig. 7(a), (b) and (c).

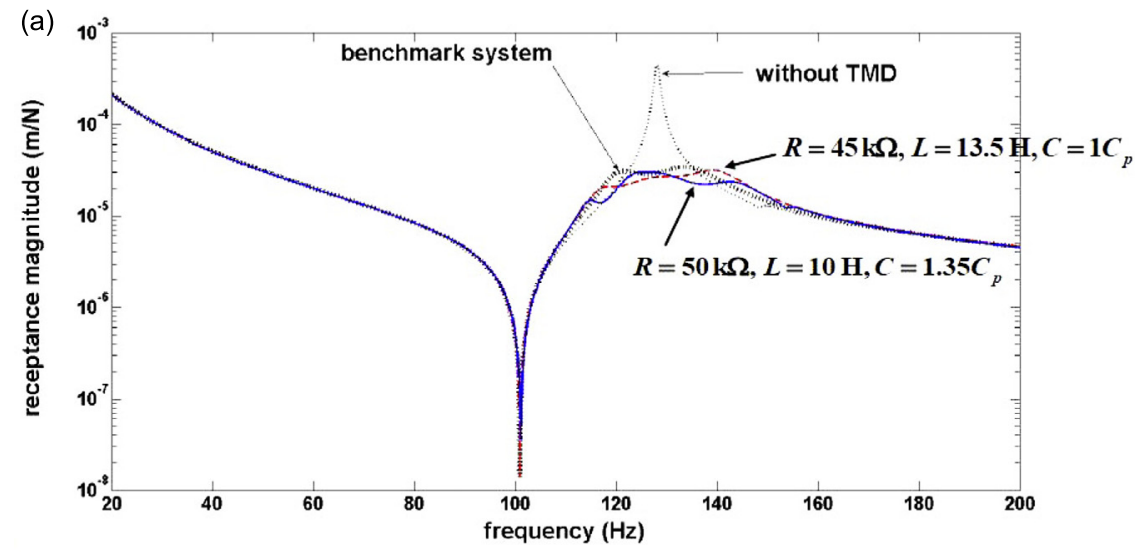

(b)

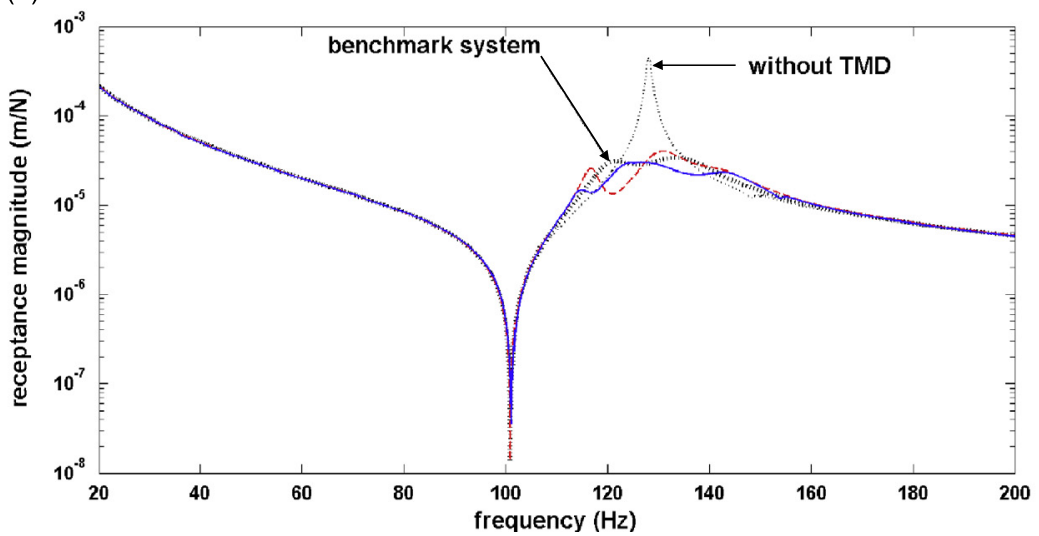

Fig. 9. Theoretical (dashed line) and experimental (solid line) receptance of host structure with TMD attached, separate circuits configuration with $C\|L\| R$ impedance: (a) at respective optimal conditions (different for theoretical and experimental); and (b) for same $R, L, C$ values, corresponding to experimental optimum $\left(R=50 \mathrm{k} \Omega, L=10 \mathrm{H}, C=1.35 C_{p}\right)$. 

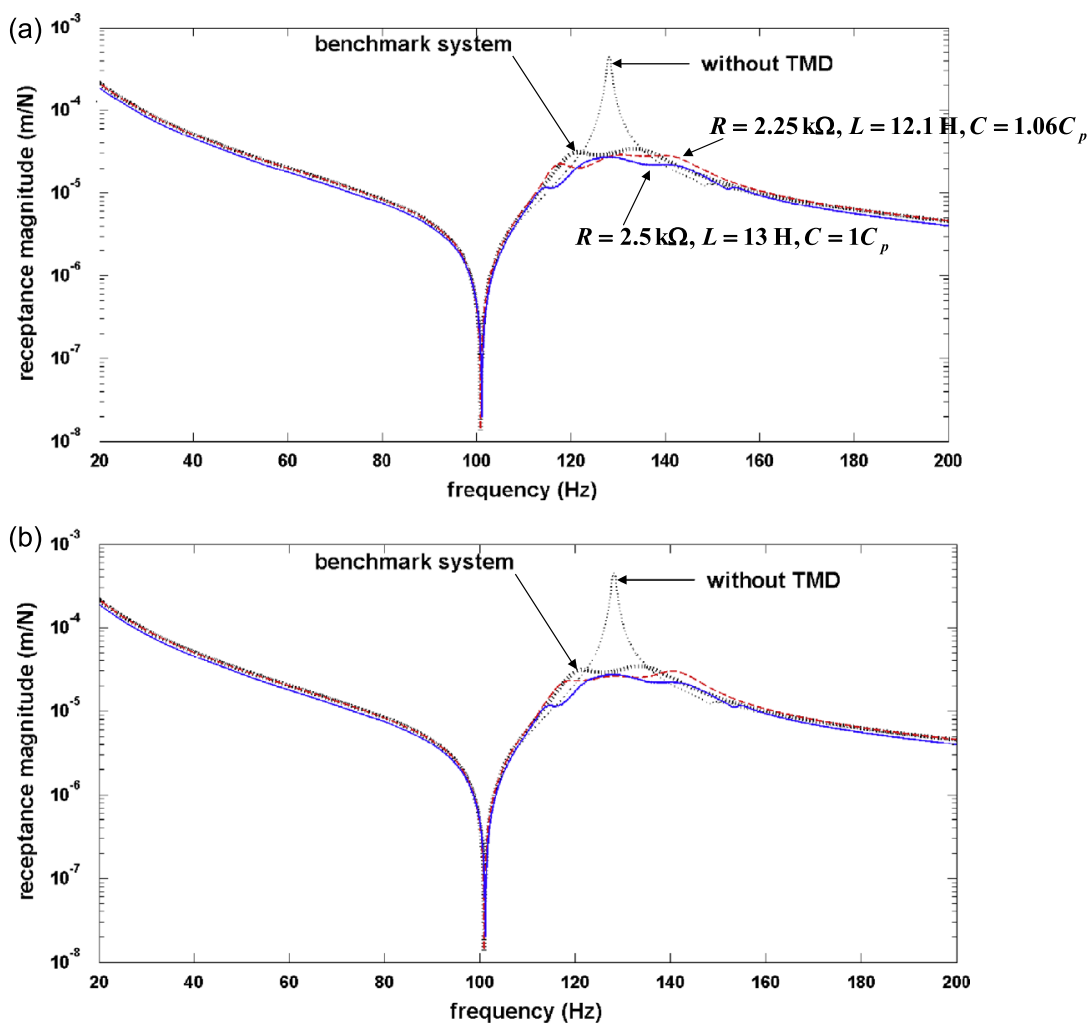

Fig. 10. Theoretical (dashed line) and experimental (solid line) receptance of host structure with TMD attached, separate circuits configuration with $C \|(L-R)$ impedance: (a) at respective optimal conditions (different for theoretical and experimental); and (b) for same $R, L, C$ values, corresponding to experimental optimum $\left(R=2.5 \mathrm{k} \Omega, L=13 \mathrm{H}, C=1 C_{p}\right)$.

conditions were reached theoretically at $R=1.8 \mathrm{k} \Omega, L=7.66 \mathrm{H}, C=1.42 C_{p}$ (Fig. 7(a)). Optimum conditions were reached experimentally at $R=1.8 \mathrm{k} \Omega, L=9 \mathrm{H}, C=1.42 C_{p}$ (Fig. 7(b)) and the response was at least as good as the benchmark. Fig. 7(c) shows that the predicted and measured responses followed the same trend as the resistance was increased to a non-optimal value of $50 \mathrm{k} \Omega$. In this latter case, due to the high impedance in the $L-R$ branch, there was virtually no attenuation in the response $r_{y_{A} F_{\text {ext }}}^{\text {host }}$ right relative to the short-circuit response in Fig. 3 due to the stiffening produced by the increased impedance.

Fig. 8(a,b) again show that optimal conditions resulted in a broad peak in the voltage FRF over the region of the targeted mode, which disappeared as the conditions were made significantly non-optimal (Fig. 8(c)).

\subsection{Separate circuits}

Figs. 9 and 10 respectively refer to the separate circuits configurations with $C\|L\| R, C \|(L-R)$ impedances in each circuit. Figs. 9(a) and 10(a), show that a response that was at least as good as the benchmark was achievable experimentally, as well as theoretically. The experimental optimal parameters were approximately in the region of the predicted ones. However, as in previous results, the response was somewhat sensitive to these differences in theoretical and experimental optimal parameters (Figs. 9(b) and 10(b)). It is also noted that, as in the single circuit case, the optimal resistance required for the $C \|(L-R)$ impedance was an order of magnitude smaller than that required for the $C\|L\| R$.

\subsection{Summary of results}

For the case studied, both theory and experiment showed that the most convenient and economical circuit configuration was the single-circuit configuration with $C \|(L-R)$ impedance since it required the smallest optimal resistor and inductor (and only one of each, Fig. 7(a,b)). As reasoned in [5], although the overall optimum R, $L, C$ values are dependent on the host structure, one would expect this conclusion to be the norm for the following reasons: a single circuit requires half the number of components; the additive effect of the bimorph internal capacitances in a single circuit tends to reduce the size of the required inductor; having $L$ in series with $R$ increases the overall impedance, reducing the required value of $R$. However, in the absence of a quantitative universal conclusion, it is prudent to consider all four circuit options for a given application prior to choosing the most appropriate configuration [5]. 


\section{Conclusions}

This paper has experimentally validated the novel concept of utilising a PVEH beam as a TMD in order to suppress a particular vibration mode of a generic host structure over a broad band of excitation frequencies. This device comprised a pair of bimorphs shunted by resistor, capacitor, inductor circuitry. The optimal damping required by this TMD was generated by the PVEH effect of the bimorphs. The benchmark for both measured and predicted performance was established by adapting Den Hartog's classical theory to a TMD in the form of a beam device. Just as predicted by the theory, the experimental results demonstrated that the ideal degree of vibration attenuation could be achieved through appropriate tuning of the circuitry using an electromechanical TMD beam device whose effective mass was less than 2 percent of the equivalent mass of the example host structure at the targeted mode.

The proposed dual PVEH/TMD beam device combines the relative advantages of the classical (mechanical) TMD and the shunted piezoelectric patch (electrical vibration absorber). Additionally, it holds the potential of simultaneous energy storage, as demonstrated by predicted and measured voltage FRFs, which showed a broad peak over the region of the targeted mode. However, for such a potential to be realised, a means of energy storage has to be introduced. This latter development is the subject of future research, using the work in [15] as a basis. Another area of possible future research is the implementation of the electrical side of the proposed TMD through synthetic circuits which, apart from eliminating the use of bulky inductor boxes, facilitates online electrical tuning [12].

\section{References}

[1] A.H. von Flotow, A.H. Beard, D. Bailey, Adaptive tuned vibration absorbers: tuning laws, tracking agility, sizing, and physical implementations, Proceedings of the Noise-Coneference 94, Florida, USA, 1994, pp. 81-101.

[2] P. Bonello, K.H. Groves, Vibration control using a beam-like adaptive tuned vibration absorber with an actuator-incorporated mass element, Proceedings of the IMechE, Part C: Journal of Mechanical Engineering Science 223 (7) (2009) 1555-1567.

[3] M. Kidner, M.J. Brennan, Improving the performance of a vibration neutraliser by actively removing damping, Journal of Sound and Vibration 221 (4) (1999) 587-606.

[4] D. Hartog, Mechanical Vibrations, Mc-Graw Hill, New York, 1956.

[5] P. Bonello, S. Rafique, R. Shuttleworth, A theoretical study of a smart electromechanical tuned mass damper beam device, Smart Materials and Structures 21 (2012) 125004.

[6] I. Saidi, A.D. Mohammed, E.F. Gad, J.L. Wilson, N. Haritos, Optimum design for passive tuned mass dampers using viscoelastic materials, Australian Earthquake Engineering Society Conference (AEES2007), Wollongong, Australia, 2007.

[7] G.M. Goethchius, Constrained layer viscoelastic laminate tuned mass damper and method of use, US Patent Application Publication no. US 2006/ 0169557 A1, 2006.

[8] C.H. Park, Dynamics modelling of beams with shunted piezoelectric elements, Journal of Sound and Vibration 268 (1) (2003) 115-129.

[9] N.W. Hagood, A. Von Flotow, Damping of structural vibrations with piezoelectric materials and passive electrical networks, Journal of Sound and Vibration 146 (2) (1991) 243-268.

[10] J. Hollkamp, T.F. Starchville, Self-tuning piezoelectric vibration absorber, Journal of Intelligent Material Systems and Structures 5 (4) (1994) 559-566.

[11] H.H. Law, Characterization of mechanical vibration damping by piezoelectric materials, Journal of Sound and Vibration 197 (4) (1996) $489-513$.

[12] A.J. Fleming, S.O. Reza Moheimani, Optimization and implementation of multimode piezoelectric shunt damping systems, IEEE/ASME Transactions on Mechatronics 7 (1) (2002) 87-94.

[13] A.J. Fleming, S.O. Reza Moheimani, Control orientated synthesis of high-performance piezoelectric shunt impedances for structural vibration control, IEEE Transactions on Control Systems Technology 13 (1) (2005) 98-112.

[14] P. Bonello, S. Rafique, Modeling and analysis of piezoelectric energy harvesting beams using the dynamic stiffness and analytical modal analysis methods, Transactions of the ASME, Journal of Vibration and Acoustics 133 (1) (2011) 011009.

[15] P. Dalzell, P. Bonello, Analysis of an energy harvesting piezoelectric beam with energy storage circuit, Smart Materials and Structures 21 (2012) 105029.

[16] R.L. Harne, Concurrent attenuation of, and energy harvesting from, surface vibrations: experimental verification and model validation, Smart Materials and Structures 21 (2012) 035016.

[17] D.J. Ewins, Modal Testing: Theory, Practice, and Application, 2nd ed. Research Studies Press, Baldock, 2000.

[18] S. Rafique, P. Bonello, Experimental validation of a distributed parameter piezoelectric bimorph cantilever energy harvester, Smart Materials and Structures 19 (9) (2010) 094008.

[19] A. Erturk, D.J. Inman, An experimentally validated bimorph cantilever model for piezoelectric energy harvesting from base excitations, Smart Materials and Structures 18 (2) (2009) 025009.

[20] Matlab Optimisation Toolbox, User's Guide, The MathWorks, Inc., Natick, Massachusetts, 2011, p. 717. 